\title{
The way forward
}

\author{
Leon Strous \\ De Nederlandsche Bank \\ Amsterdam, The Netherlands \\ strous@iaehv.nl
}

Abstract: The closing session of the working conference was a panel discussion. Panel members were in (alphabetical order): Eric Gheur (Galaxia, Belgium), William List (Wm. List \& Co, UK), Frank Piessens (Katholieke Universiteit Leuven, Belgium), Bhavani Thuraisingham (MITRE Corporation / National Science Foundation, USA). The panel addressed issues raised during the conference and worthwhile pursuing in the next event.

Key words: data quality, standards, software engineering, safety critical systems

\section{INTRODUCTION}

In preparing the panel session, some topics were identified where members from both academia and industry definitely could make a worthwhile contribution. The theme that we had in mind was "Integrity and Internal Control in Information Systems: old and new challenges". The outcome of this panel session is to serve as input for the next IICIS conference.

To address the current problems in the area of integrity and internal control a number of questions were prepared. For example:

- Has the problem become more complex because the systems for which we want to develop integrity and internal control methods have become more complex? 
- Have the systems we are interested in actually become more complex, e.g., current banking and e-business environments?

- Do we still have the right methods and tools to do the job?

- What are the specific problems (regarding these new types of systems)?

- Do we have a sufficient understanding of the systems we are always talking about?

- Do existing standards help in dealing with the problems?

- What are emerging techniques that could help solving the problem? For example, do data mining and knowledge discovery techniques provide security personnel with appropriate tools?

- What are the systems people from industry are currently struggling with regarding the issue of integrity and internal control? What (new) achievements does academia have to offer?

\section{DISCUSSION}

Thanks to the lively and interactive presentations during the two days of the conference, sufficient discussion topics had already been raised during the days. The panel session therefore smoothly continued the discussions started earlier.

One of the major issues of concern was the question how to sell integrity and security to management. Here, the participants and panel members pointed out an obvious relationship with internal control and the recent standards in this area, like COBIT (as explained also during the tutorial). It was considered helpful if the auditors in their management letters and statements could include integrity and security issues. But also security professionals should try to use at least some of the internal control language which management often understands better than security terminology because the link to business processes is closer. Another option to help "selling" the good cause is to coordinate with areas like data quality which might be more appealing to management.

A problem encountered by vendors of security software that monitors the activity within the computer and its interaction with the outside world, is the fact that solutions embedded in software are not always accepted in a number of countries or in a number of organizations. The reasons for this can be found in social / cultural habits and/or in legal restrictions. Many employees find it threatening to be monitored (either literally or figuratively), it gives them a "big brother is watching you" feeling. If not for legal reasons, this is again a matter of how to sell your cause. Monitoring 
can also be beneficial for employees in cases where their innocence can be proved if fraud is suspected. The question is whether monitoring can contribute to the integrity of data and systems or whether this technique only / mainly serves other purposes.

The conclusion from the discussion about the first two topics is that further work needs to be done in investigating how integrity (and other security) solutions can be presented as an integral part of a system, business process or even enterprise architecture.

Moving towards the question whether standards can help to solve the above problems, the discussion focussed on a few standards currently available. These varied from baseline controls (ISO/IEC 17799) and security management guidelines (ISO 13335) to software engineering standards that include integrity aspects (ISO 15026). Besides the discussion about well known problems (e.g., it takes too long to develop standards, often compromise which means not the best standard) there was also a clear consensus about the fact that there are many standardization efforts, both through formal standards bodies and industry fora, which very often lack knowledge about each others activities, let alone cooperation to draft standards that cover more than one (small) area. Also the combination of technical and non-technical standards is an issue to look into.

Some of the questions listed in the introduction were not specifically addressed during the panel session, but were briefly discussed during other presentations. Examples of this are the questions "What are the systems people from industry are currently struggling with regarding the issue of integrity and internal control? What (new) achievements does academia have to offer?". In the presentation about modern banking systems for example, the increasing complexity of online real-time distributed banking applications was demonstrated and a new paradigm for controls in the finance and banking industry was proposed. It is a challenge for researchers and vendors to come up with useful solutions for this paradigm.

\section{CONCLUSION}

The panel chair concluded from the discussion during the panel session, the discussions during the presentations and the discussions outside the conference room that the work of working group 11.5 needed continuation. It was a general feeling that the chosen formula of different types of 
contributions was a very rewarding one for all participants. The conference should take place annually rather than bi-annually.

For the next event, submissions should be invited that address the questions listed in the introduction of this section. To ensure that progress is made in the area of integrity and internal control, it is essential to continue the dialogue between:

- Disciplines: data quality, software engineering, safety critical systems, standards, etc.;

- Professions: researchers, practitioners, vendors, standards developers, customers. 